\section{Keeping on top of inflammation}

Topoisomerases are ubiquitous enzymes that regulate the winding of DNA and thereby serve important roles in controlling gene expression. In Science, Marazzi and colleagues show that the topoisomerase Top1 positively regulates inflammation after challenge with microbial products, viruses or bacteria. Inhibition or knockdown of Top1 leads to lower expression of proinflammatory cytokines and interferon-stimulated genes and resistance to lethal inflammation triggered by viral or bacterial infection as well as by endotoxin challenge. Top1 seems to aid the expression of a highly select subset of genes encoding proinflammatory products—specifically those that require nucleosome remodeling for their activation. Mechanistically, Top1 facilitates the presence of the RNA polymerase RNAPII at specific genes that encode inflammatory products and thereby influences their expression. Clinically approved inhibitors of Top1 are already used in treating cancer but could potentially also be used for the management of harmful hyper-inflammation.

ZF

Science (28 April 2016) doi:10.1126/science.aad7993

\section{Coping with stress}

Stress has well-appreciated deleterious effects on the immune system. In the Proceedings of the National Academy of Sciences, Lowry and colleagues demonstrate that exposure of mice to heat-killed Mycobacterium vaccae, a free-living bacteria with immunomodulatory properties, can promote resilience in a mouse model of psychosocial stress. Vaccination of mice with $M$. vaccae produces a relatively long-lasting benefit in coping with stress. In addition to the behavioral effects of exposure to $M$. vaccae, mice also show alterations in serotonergic systems in the brain and increased density of microglia in brain regions associated with fear responses. Stress also exacerbates experimental models of inflammatory bowel disease. Accordingly, M. vaccae also ameliorates stress-enhanced inflammatory bowel disease. The beneficial effects of $M$. vaccae on stress are mediated at least in part by its ability to generate and/or support the function of regulatory T cells.

ZF

Proc. Natl. Acad. Sci. USA (16 May 2016) doi:10.1073/pnas.1600324113

\section{Neuronal antibody access}

Antibodies are required for full protection against neurotrophic viruses, but how antibodies gain access to neuronal tissues is unclear. In Nature, lijima and Iwasaki show that antigen-specific $\mathrm{CD}^{+}$memory $\mathrm{T}$ cells $\left(\mathrm{T}_{\text {mem }}\right.$ cells) facilitate the entry of IgG2 antibodies into the dorsal root ganglia during infection with herpes simplex virus type 2 and their entry into other neuronal sites after infection with vesicular stomatitis virus. Circulating antibodies to herpes simplex virus type 2 provide protection in the innervating neurons but not in vaginal tissues. Local production of interferon- $\gamma$ (IFN- $\gamma$ ) by the infiltrating $\mathrm{CD}^{+}{ }^{+} \mathrm{T}$ cells modulates vasculature permeability, which allows access of protective antibodies to infected neurons to limit viral spreading. Curiously, the route of immunization also influences whether protective responses can be elicited. Whether this last finding is due to eliciting a particular antibody isotype or proper homing properties of the $\mathrm{CD}^{+} \mathrm{T}$ cells remains unknown.

Nature (18 May 2016) doi:10.1038/nature17979

\section{The tryptophan link}

Microbiota-derived products function as immunoregulatory metabolites. In Nature Medicine, Quintana and colleagues show that dietary tryptophan is metabolized by the gut microbiota into agonists of the transcription factor AhR that act on astrocytes to limit inflammation of the central nervous system. Experimental autoimmune encephalomyelitis in mice induces a strong type I interferon response as well as upregulation of Ahr mediated by the transcription factors STAT1-STAT2 in astrocytes. Astrocyte-specific knockdown of the interferon receptor Ifnarl or Ahr induces the expression of genes encoding pro-inflammatory products in astrocytes, microglia and Ly6 $\mathrm{C}^{\text {hi }}$ monocytes and increases the recruitment of inflammatory monocytes to the central nervous system. Dietary deficiency in tryptophan worsens experimental autoimmune encephalomyelitis in mice, a process linked to AhR expression in astrocytes. Patients with multiple sclerosis have lower expression of tryptophanderived AhR ligands in the serum than that of healthy control subjects, which suggests that deficiency in AhR agonists linked to metabolism, diet or the microbiota can contribute to the pathogenesis of multiple sclerosis.

Nat. Med. (9 May 2016) doi:10.1038/nm.4106

\section{Nucleocytoplasmic danger signals}

The release of danger signals from damaged or infected cells leads to the recruitment of leukocytes to the inflammed site. In Cell, Enyedi et al. show that tissue hypo-osmolarity can trigger the release of danger signals. Osmotic swelling induces biophysical changes in the nuclear envelope, including alterations in the nuclear lamin and actin cytoskeleton, that cause translocation and activation of the phospholipase enzyme PLA $A_{2}$. Both nuclear swelling and calcium flux are required for activation of PLA $\mathrm{A}_{2}$. This response leads to release of archidonic acid from the nuclear membrane and its oxidation to proinflammatory lipid mediators by 5 -lipoxygenase. These findings suggest that nuclear-membrane tension acts as a sensor of osmolarity that, when perturbed, is perceived as a danger signal and results in the release of chemoattractants.

$L A D$

Cell 165, 1160-1170 (2016)

\section{Acetate for memory}

Systemic concentrations of acetate increase in metabolic-stress conditions, such as starvation and pathogen infection. In Immunity, Hess and colleagues show that increased concentrations of extracellular acetate during bacterial infection are required for optimal responses of $\mathrm{CD}^{+} \mathrm{T}_{\text {mem }}$ cells. Bacterial infection in mice triggers the release of acetate into the systemic circulation, and in vitro exposure of $\mathrm{T}_{\text {mem }}$ cells to acetate concentrations similar to those found during early infection enhances their production of IFN- $\gamma$, glycolytic reserve and spare respiratory capacity. Exogenous acetate is metabolized into acetyl-CoA and promotes acetylation of the glycolytic enzyme GAPDH in $\mathrm{T}_{\text {mem }}$ cells. This post-translational modification is required for GAPDH's enzymatic function and IFN- $\gamma$ production. In vivo treatment with acetate increases the glucose metabolism and production of IFN- $\gamma$ in $\mathrm{CD}^{+}$ $\mathrm{T}_{\text {mem }}$ cells during reactivation, while the transfer of acetate-exposed $\mathrm{CD}^{+} \mathrm{T}_{\text {mem }}$ cells controls bacterial infection better than the transfer of untreated cells does.

Immunity (17 May 2016) doi:10.1016/j.immuni.2016.03.016 